\title{
GENERALIZED DERIVATIVES AND THE DE LA VALLEE POUSSIN DERIVATIVE ${ }^{1}$
}

\section{KASSIMATIS}

Let $V_{n}\left[f: p_{1}, \cdots, p_{n+1}\right]$ denote the $n$th divided difference of $f$ relative to the distinct points $p_{1}, \cdots, p_{n+1}$, where $f$ is a real valued continuous function over the interval $(a, b)$ and $p_{i} \in(a, b), i=1$, $\cdots, n+1$.

If

$$
\lim _{h \rightarrow 0} n ! V_{n}\left[f: x+x_{1} h, \cdots, x+x_{n+1} h\right]
$$

exists and is finite, it is called, following Denjoy [1, p. 305], the $n$th $E$-generalized derivative of $f$ at $x, f_{n, E}(x)$, where $E=\left\{x_{1}, \cdots, x_{n+1}\right\}$. If $f_{n, E}(x)$ is independent of the choice of $E \subset S$ for a subset $S$ of the reals, we will call it the $n$th $S$-generalized derivative $f_{n, S}(x)$.

If

$$
f(x+t)=a_{0}+a_{1} t+\cdots+a_{n} t^{n} / n !+o\left(t^{n}\right)
$$

where the numbers $a_{0}, a_{1}, \cdots, a_{n}$ depend on $x$ only, then $a_{n}$ will be called, following Marcinkiewicz and Zygmund [5, p. 1], the nth de la Vallee Poussin derivative of $f$ at $x, f_{(n)}(x)$.

Denjoy has shown $\left[1\right.$, p. 289] that the existence of $f_{(n)}(x)$ implies that of $f_{n, S}(x)$ for $S$ arbitrary. In a previous paper [4] the author has obtained a converse of the above Denjoy theorem. The purpose of the present paper is to establish a more general form of this converse theorem.

Let $f$ and $S$ be as above. Denote by $S_{j+1}$ any $(j+1)$-tuple of distinct points of $S, j=1, \cdots, n-1$. For $x \in(a, b)$ and $s_{i} \in S$, set $y_{i}=x+s_{i} h$, $i=1, \cdots, n+1$.

Theorem. Suppose that $f_{n, s}(x)$ exists. If

(i) $f_{j, s_{+1}}(x)$ exists for all $j=1, \cdots, n-1$,

(ii) $\lim _{h \rightarrow 0}\left\{\left(y-y_{n+1}\right) V_{n+1}\left[f: y_{1}, \cdots, y_{n+1}, y\right]\right\}=\theta(x, y-x)$ where $\theta(x, y-x) \rightarrow 0$ as $y \rightarrow x$, then $f_{(n)}(x)$ exists and $f_{(n)}(x)=f_{n, S}(x)$.

Proof. By [3, Corollary 3] we obtain the existence of $f_{1, s}(x)$, $\cdots, f_{n-1, S}(x)$. Consequently, $f_{1, E_{2}}(x), \cdots, f_{n, E_{n+1}}(x)$ exist, where $E_{i}=\left\{s_{1}, \cdots, s_{i}\right\}$ for $i=1, \cdots, n+1$, and the result follows by [4, Theorem].

Received by the editors July 26, 1965.

1 This research was supported by the National Research Council Grant MCA-31. 
Remarks. (i) As mentioned earlier, Denjoy has shown that the existence of $f_{(n)}(x)$ implies that of $f_{n, S}(x)$ for $S$ arbitrary. Since the existence of $f_{(n)}(x)$ implies by (1) that of $f_{(1)}(x), \cdots, f_{(n-1)}(x)$, it follows that the existence of $f_{(n)}(x)$ implies the first condition of the above theorem.

(ii) The existence of $f_{(n)}(x)$ implies also the second condition of the above theorem. Indeed in this case, the expression $f(x+t)+o\left(t^{n}\right)$ is by (1) a polynomial of degree at most $n$ and it follows by [2, Theorem 2.1] that

$$
0=V_{n+1}\left[f(x+t)+o\left(t^{n}\right): s_{1} h, \cdots, s_{n+1} h, y-x\right] .
$$

Hence,

$$
\begin{aligned}
V_{n+1}\left[f(t): x+s_{1} h,\right. & \left.\cdots, x+s_{n+1} h, y\right] \\
& =V_{n+1}\left[o\left(t^{n}\right): s_{1} h, \cdots, s_{n+1} h, y-x\right] .
\end{aligned}
$$

Further, evaluating the divided difference we get:

$$
\begin{aligned}
\left(y-y_{n+1}\right) V_{n+1}\left[o\left(t^{n}\right): s_{1} h, \cdots, s_{n+1} h, y-x\right] & \\
= & \frac{\left(y-y_{n+1}\right) o\left((y-x)^{n}\right)}{\left(y-y_{1}\right) \cdots\left(y-y_{n+1}\right)} \\
& \quad-\sum_{i=1}^{n+1} \frac{\left(y-y_{n+1}\right) o\left(s_{i}^{n} h^{n}\right)}{h^{n}\left(y-y_{i}\right)\left\{\left(u-s_{1}\right) \cdots\left(u-s_{n+1}\right)\right\}_{u=s_{i}}^{\prime}} .
\end{aligned}
$$

When $h \rightarrow 0$, we obtain

$$
\lim _{h \rightarrow 0}\left\{\left(y-y_{n+1}\right) V_{n+1}\left[o\left(t^{n}\right): s_{1} h, \cdots, s_{n+1} h, y-x\right]\right\}=\theta(x, y-x) .
$$

The result follows from relation (2).

(iii) The following example shows that the first condition of the above theorem cannot alone ensure the existence of $f_{(n)}(x)$. Consider the continuous function $f(x)=x|x|$. Let $n=2, x=0, s_{1}=-1, s_{2}=0$, $s_{3}=1$. Evaluating the generalized derivatives we find:

$$
f_{1,\{-1,0\}}(0)=0 ; \quad f_{2,\{-1,0,1\}}(0)=0 .
$$

For $y>0$, we find that

$$
\lim _{h \rightarrow 0}\left\{(y-h) V_{3}[x|x|:-h, 0, h, y]\right\}=1 \neq \theta(0, y-0) .
$$

The de la Vallée Poussin derivative $f_{(2)}(0)$ does not exist because otherwise we must have

$$
t|t|=f_{(1)}(0) t+f_{(2)}(0) t^{2} / 2+o\left(t^{2}\right),
$$


whence $f_{(1)}(0)=o\left(t^{2}\right)=0$. Therefore

$$
t|t|=f_{(2)}(0) t^{2} / 2 \text {. }
$$

From this relation we obtain $f_{(2)}(0)=2$ when $t>0$, and $f_{(2)}(0)=-2$ when $t<0$. Since $f_{(2)}(0)$ here depends on $t$, the second de la Vallée Poussin derivative of $x|x|$ does not exist at the origin.

\section{REFERENCES}

1. A. Denjoy, Sur l'intégration des coefficients différentiels d'ordre superieur, Fund. Math. 25 (1935), 273-326.

2. C. Kassimatis, Certain properties of the generalized integral of a finite Riemann derivative, Trans. Roy. Soc. Canad. Ser. III 53 (1959), 31-36.

3. - On generalized derivatives, Studia Math. 25 (1965), 369-371.

4. - On the de la Vallée Poussin derivative, Proc. Amer. Math. Soc. 16 (1965), 1171-1172.

5. J. Marcinkiewicz and A. Zygmund, On the differentiability of functions and summability of trigonometrical series, Fund. Math. 26 (1936), 1-43.

WAyne State UNIVERSity 